\title{
Data-Driven Inventory Management and Dynamic Pricing Competition on Online Marketplaces
}

\author{
Rainer Schlosser, Carsten Walther, Martin Boissier, Matthias Uflacker \\ Hasso Plattner Institute, University of Potsdam, Germany \\ \{firstname.lastname\}@hpi.de
}

\begin{abstract}
Online markets are characterized by competition and limited demand information. In E-commerce, firms compete against each other using data-driven dynamic pricing and ordering strategies. Successfully managing both inventory levels as well as offer prices is a highly challenging task as (i) demand is uncertain, (ii) competitors strategically interact, and (iii) optimized pricing and ordering decisions are mutually dependent. Currently, retailers lack the possibility to test and evaluate their algorithms appropriately before releasing them into the real world. To study joint dynamic ordering and pricing competition on online marketplaces, we built an interactive simulation platform. To be both flexible and scalable, the platform has a microservice-based architecture and allows handling dozens of competing merchants and streams of consumers with configurable characteristics. Further, we deployed and compared different pricing and ordering strategies, from simple rule-based ones to sophisticated datadriven strategies which are based on state-of-the-art demand learning techniques and efficient dynamic optimization models.
\end{abstract}

\section{Joint Ordering and Pricing Competition}

E-commerce has become highly dynamic and competitive. Merchants are able to automatically adjust prices to react to changing market situations within milliseconds, [Greenwald and Kephart, 1999]. Similarly, they can flexibly reorder items taking into account (i) estimated demand, (ii) inventory holding costs, and (iii) delivery times.

To derive effective pricing and ordering decisions over time is challenging considering that numbers of combinations are enormous, demand is uncertain, and markets are steadily changing (cf. [Tsai and Hung, 2009], [Adida and Perakis, 2010], [Chen and Chen, 2015]). Moreover, pricing and ordering strategies mutually affect each other [Rajan et al., 1992].

Simulating the performance of automated ordering and pricing strategies is important as testing is potentially hazardous when done in production. To the best of our knowledge, however, flexible simulation platforms that allow evaluating specific strategies under competition do not exist.
Existing platforms, e.g., [Kephart et al., 2000], [DiMicco et al., 2003], are limited in their capabilities: Simulations run on a single machine, offer a limited set of consumer behaviors, simulate solely short sales horizons, and pricing updates or orders are restricted to predefined discrete points in time.

We built a continuous time framework (mimicking production marketplaces such as Amazon or eBay [Boissier $e t$ al., 2017; PriceWars, 2018]) to simulate dynamic pricing and ordering under competition. Our setup supports customers with heterogeneous buying behaviors. The competitors' offers include multiple dimensions such as price and quality.

The platform allows an arbitrary number of merchants to compete simultaneously. Each merchant can run his preferred repricing and ordering strategy to adjust prices on the marketplace and to order products, respectively. Due to the strategic interaction of competing merchants' price reactions market situations steadily change. Simulating streams of customer decisions allows generating realized sales events and the firms' sales revenues. The firms' inventory levels, their holding costs as well as their ordering costs are determined by their ordering strategies. Finally, the user can easily study the complex interplay of ordering and repricing strategies and, most importantly, their performance (e.g., short and long-term profits).

Our system supports self-adapting learning strategies. The platform logs each interaction such as orders, price updates, stock-outs, new offers, sales, etc. This historical data - which is defined as partially observable (sales are private knowledge) - is requested and numerically analyzed by data-driven merchants. Various state-of-the-art machine learning approaches can be applied to quantify how demand (i.e., sales probabilities) is affected by a merchant's pricing decisions.

Further, merchants are able to deploy optimization models [Transchel and Minner, 2009; Schlosser and Boissier, 2017; Yabe et al., 2017]. which are calibrated by estimated sales probabilities to compute optimized data-driven pricing and ordering strategies. In this context, it can be even tried to learn about competitors' strategies in order to take anticipated price reactions into account.

Finally, our framework also allows controlling and measuring the influence of (i) the customers' buying behavior, (ii) price adjustment frequencies, as well as (iii) the exit or entry of competitors on a strategy's performance. In addition, different demand learning techniques and optimization approaches can be compared regarding their accuracy and efficiency. 
Proceedings of the Twenty-Seventh International Joint Conference on Artificial Intelligence (IJCAI-18)

\begin{tabular}{lcccc}
\hline Merchant & Profit & Revenue & Holding Costs & Order Costs \\
\hline Data-Driven & 534.17 & 3547.00 & 212.83 & 2800.00 \\
Merchant A & 315.12 & 4063.80 & 363.80 & 3385.00 \\
Merchant B & 492.50 & 3844.80 & 152.30 & 3200.00 \\
\hline
\end{tabular}

Table 1: Comparison of data-driven and rule-based strategies.

\section{Platform Description and Implementation}

The platform is built with a microservice-based architecture for scalability and flexibility. Each service implements one business artifact, whereby services can be scaled out for large simulations. By having separated services, additional components can be added during running simulations at any time.

Merchants can be easily added to the simulation or updated as long as they confirm to the HTTP/REST interface of the platform [Serth et al., 2017]. Merchants update their products' prices based on the current market situation which they can request at any time. Arbitrary strategies, e.g., rule-based or data-driven strategies, can be applied. Data of observed market situations as well as a merchant's sales data can be used to estimate sales probabilities using various machine learning techniques (cf. [Schlosser and Boissier, 2018]).

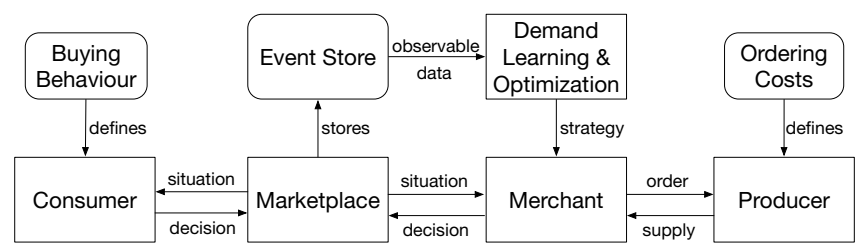

Figure 1: Depiction of the platform's components and their interaction in a dynamic pricing scenario with inventory replenishment.

The centre of the simulation is the marketplace which manages all product offers, cf. Figure 1. Offers include price and quality. The marketplace is the access point for the consumer component which creates a random stream of interested customers. Any customer choice behavior can be defined. The decision whether a customer buys a product and which offer is chosen, is modelled by probabilities that can depend on all parameters of the current market situation.

The event store logs platform events (price adjustments, sales, etc.) and provides CSV files for data-driven merchants. The producer provides products ordered by the merchants. The merchants regularly request current market situations and decide on price updates and orders. For optimized well-matched pricing and ordering decisions they can apply demand learning to estimate sales probabilities to be used in (dynamic) optimization models. In our implementation, we applied efficient dynamic programming techniques.

Finally, the HTML-based front end enables the user to configure the customer behavior, the merchants' strategy setup, as well as cost parameters (fixed/variable ordering costs, holding cost rate [Schlosser, 2015]). The front end further allows observing prices (see Figure 2(a)), inventories (see Figure 2(b)), and profits over time. The strategies' short-term and long-term performances are measured by different KPIs, including profits, revenues, holding costs, ordering costs, etc. (see Table 1).
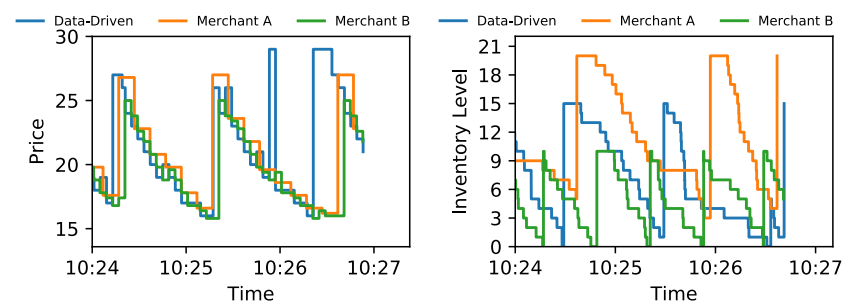

(a) Competitors' price trajectories (b) Competitors' inventory levels

Figure 2: Competitors' pricing and ordering decisions over time.

\section{Results}

The platform allows simulating strategic interaction of rulebased and advanced data-driven strategies in different market scenarios characterized by product portfolios, customer behaviors, oligopoly settings, and cost definitions.

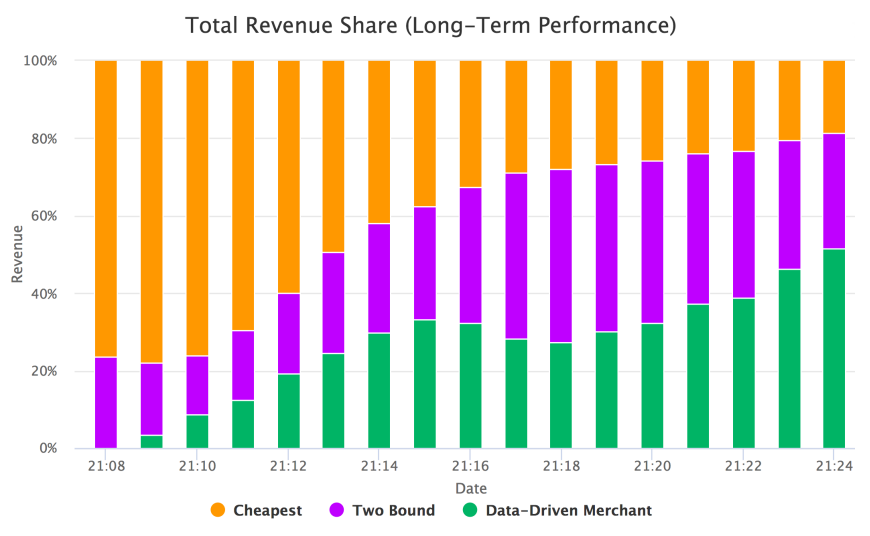

Figure 3: Screenshot of the demonstration, showing total revenue share for a learning data-driven merchant and two competitors.

We found that fully automated data-driven strategies - combined with efficient dynamic programming optimization approaches - vastly outperform rule-based ones after a sufficiently large data set has been gathered. It can also be studied to which extent jointly optimized pricing and ordering strategies outperform different combinations of single ordering and pricing benchmark strategies. Moreover, the platform can be used to study short-term as well as long-term performance of self-adapting strategies that iteratively improve over time.

\section{Conclusion and Future Work}

The presented dynamic market simulation is a distributed and scalable platform mimicking real-world e-commerce applications. With this toolkit, both practitioners and researchers can directly investigate various adaptive pricing and ordering strategies under competition and develop, test, and evaluate own approaches. Furthermore, our model can be easily extended in several ways: (i) further offer dimensions (ratings, shipping time, etc. [Kachani and Shmatov, 2010]), (ii) the consideration of perishable products, and (iii) substitution effects between different products [Ito and Fujimaki, 2017]. 


\section{References}

[Adida and Perakis, 2010] Elodie Adida and Georgia Perakis. Dynamic pricing and inventory control: Uncertainty and competition. Operations Research, 58(2):289-302, 2010.

[Boissier et al., 2017] Martin Boissier, Rainer Schlosser, Nikolai Podlesny, Sebastian Serth, Marvin Bornstein, Johanna Latt, Jan Lindemann, Jan Selke, and Matthias Uflacker. Data-driven repricing strategies in competitive markets: An interactive simulation platform. In Proceedings of the Conference on Recommender Systems, RecSys, pages 355-357, 2017.

[Chen and Chen, 2015] Ming Chen and Zhi-Long Chen. Recent developments in dynamic pricing research: Multiple products, competition, and limited demand information. Production and Operations Management, 24(5):704-731, 2015.

[DiMicco et al., 2003] Joan Morris DiMicco, Pattie Maes, and Amy Greenwald. Learning curve: A simulation-based approach to dynamic pricing. Electronic Commerce Research, 3(3-4):245-276, 2003.

[Greenwald and Kephart, 1999] Amy R. Greenwald and Jeffrey O. Kephart. Shopbots and pricebots. In International Joint Conference on Artifical Intelligence, volume 1, pages 506-511, 1999.

[Ito and Fujimaki, 2017] Shinji Ito and Ryohei Fujimaki. Optimization beyond prediction: Prescriptive price optimization. In International Conference on Knowledge Discovery and Data Mining, pages 1833-1841, 2017.

[Kachani and Shmatov, 2010] Soulaymane Kachani and Kyrylo Shmatov. Competitive pricing in a multi-product multi-attribute environment. Production and Operations Management, 20(5):668-680, 2010.

[Kephart et al., 2000] Jeffrey O. Kephart, James E. Hanson, and Amy R. Greenwald. Dynamic pricing by software agents. Computer Networks, 32(6):731-752, 2000.

[PriceWars, 2018] PriceWars. Source and Documentation - Repository on GitHub: https://git.io/ pricewars. Last accessed: May 22nd, 2018.
[Rajan et al., 1992] Arvind Rajan, Rakesh, and Richard Steinberg. Dynamic pricing and ordering decisions by a monopolist. Management Science, 38(2):240-262, 1992.

[Schlosser and Boissier, 2017] Rainer Schlosser and Martin Boissier. Optimal price reaction strategies in the presence of active and passive competitors. In International Conference on Operations Research and Enterprise Systems, ICORES, pages 47-56, 2017.

[Schlosser and Boissier, 2018] Rainer Schlosser and Martin Boissier. Dynamic pricing under competition on online marketplaces: A data-driven approach. In Proceedings of the ACM SIGKDD International Conference on Knowledge Discovery and Data Mining, 2018.

[Schlosser, 2015] Rainer Schlosser. Dynamic pricing and advertising models with inventory holding costs. Journal of Economic Dynamics and Control, 57:163-181, 2015.

[Serth et al., 2017] Sebastian Serth, Nikolai Podlesny, Marvin Bornstein, Johanna Latt, Jan Lindemann, Jan Selke, Rainer Schlosser, Martin Boissier, and Matthias Uflacker. An interactive platform to simulate dynamic pricing competition on online marketplaces. In 21st IEEE International Enterprise Distributed Object Computing Conference, EDOC, pages 61-66, 2017.

[Transchel and Minner, 2009] Sandra Transchel and Stefan Minner. The impact of dynamic pricing on the economic order decision. European Journal of Operational Research, 198(3):773-789, 2009.

[Tsai and Hung, 2009] Wen-Hsien Tsai and Shih-Jieh Hung. Dynamic pricing and revenue management process in internet retailing under uncertainty: An integrated real options approach. Omega, 37(2-37):471-481, 2009.

[Yabe et al., 2017] Akihiro Yabe, Shinji Ito, and Ryohei Fujimaki. Robust quadratic programming for price optimization. In International Joint Conference on Artificial Intelligence, 2017. 